\title{
Zeaxanthin epoxidation - an in vitro approach*
}

\section{Paulina Kuczyńska1,2, Dariusz Latowski ${ }^{1 凶}$, Sylvia Niczyporuk³, Monika Olchawa-Pajor ${ }^{1}$, Peter Jahns ${ }^{3}$, Wiesław I. Gruszecki ${ }^{4}$ and Kazimierz Strzałka ${ }^{1}$}

1Department of Plant Physiology and Biochemistry, Jagiellonian University, Kraków, Poland; 2Department of Plant Physiology, Institute of Biology, Pedagogical University, Kraków, Poland; ${ }^{3}$ Plant Biochemistry, Heinrich-Heine-University Düsseldorf, Düsseldorf, Germany; ${ }^{4}$ Department of Biophysics, Maria Curie-Skłodowska University, Lublin, Poland

Zeaxanthin epoxidase (ZE) is an enzyme operating in the violaxanthin cycle, which is involved in photoprotective mechanisms. In this work model systems to study zeaxanthin (Zx) epoxidation were developed. Two assay systems are presented in which epoxidation of $\mathrm{Zx}$ was observed. In these assays two mutants of Arabidopsis thaliana which have active only one of the two xanthophyll cycle enzymes were used. The npq1 mutant possesses an active $Z E$ and is thus able to convert $Z x$ to violaxanthin $(V x)$ but the violaxanthin de-epoxidase (VDE) is inactive, so that $\mathrm{Vx}$ cannot be converted to $\mathrm{Zx}$. The other mutant, npq2, possesses an active VDE and can convert exogenous $V x$ to $Z x$ under strong light conditions but reverse reaction is not possible. The first assay containing thylakoids from $n p q 1$ and $n p q 2$ mutants of $A$. thaliana gave positive results and high efficiency of epoxidation reaction was observed. The amount of $Z x$ was reduced by $25 \%$. To optimize high efficiency of epoxidation reaction additional factors facilitating both fusion of the two types of thylakoids and incorporation of $\mathrm{Zx}$ to their membranes were also studied. The second kind of assay contained npq 1 mutant thylakoids of $A$. thaliana supplemented with exogenous $Z x$ and monogalactosyldiacylglycerol (MGDG). Experiments with different proportions of $Z x$ and MGDG showed that their optimal ratio is 1:60. In such system, due to epoxidation, the amount of $Z \mathbf{x}$ was reduced by $38 \%$ of its initial level. The in vitro systems of $Z x$ epoxidation described in this paper enable analysis some properties of the ZE without necessity of its isolation.

Key words: zeaxanthin epoxidation, xanthophyll cycle, model system, Arabidopsis thaliana mutants npq1, npq2

Received: 17 October, 2011; accepted: 01 March, 2012; available on-line: 17 March, 2012

\section{INTRODUCTION}

Violaxanthin cycle is the most common of the six xanthophyll cycle types described so far in the literature. It occurs in higher plants, mosses, lichens and some groups of algae protecting them against overexcitation (Garcia-Plazaola et al., 2007; Goss et al.,2006). Two reversible reactions occur in the violaxanthin cycle. One of them is de-epoxidation of a di-epoxide xanthophyll, violaxanthin $(\mathrm{Vx})$, by violaxanthin de-epoxidase (VDE). This enzyme is located in the thylakoid lumen and for activation requires ascorbate as a donor of protons, monogalactosyldiacylglycerol (MGDG) or another non-bilayer lipids such as, phosphatidylethanolamine (PE) and an acidic $\mathrm{pH}$ which is necessary for binding VDE to thylakoid membrane (Hager \& Holocher, 1994; Latowski et al., 2004, Jahns et al., 2009). In the reverse reaction catalysed by zeaxanthin epoxidase (ZE), zeaxanthin $(\mathrm{Zx})$, an epoxy-free pigment is converted to $\mathrm{Vx}$. ZE, which is located on the stromal side of thylakoids, is a monooxygenase epoxidizing 3 -hydroxy $\beta$-ionone rings of xanthophylls in 5,6 position. NADPH, FAD, molecular oxygen and $\mathrm{pH} 7.5$ are required for $\mathrm{Zx}$ epoxidation (Siefermann \& Yamamoto, 1975; Büch et al., 1995). There exist model systems to study $\mathrm{Vx}$ de-epoxidation with the use of isolated enzyme (Yamamoto \& Higashi, 1978; Latowski et al., 2002). Such approach in the case of Zx epoxidation is difficult because the respective enzyme has not been isolated and purified so far. In this paper two kinds of model systems for studies on $\mathrm{Zx}$ epoxidation in vitro are presented for the first time. In both systems reduction in the $\mathrm{Zx}$ amount was observed. In these assays two mutants of Arabidopsis thaliana which have active only one of the two xanthophyll cycle enzymes were used. The first one, $n p q 1$, produces active $\mathrm{ZE}$ and it converts Zx to $\mathrm{Vx}$, while VDE is inactive and photoprotection by xanthophyll cycle mechanism is not observed (Havaux et al., 2000). The other mutant, npq2, possesses active $\mathrm{VDE}$ and it is able to convert exogenous $\mathrm{Vx}$ to $\mathrm{Zx}$ under strong light but reverse reaction is not possible. This mutant can accumulate $\mathrm{Zx}$ and it was used as a source of this pigment in our experiments.

\section{MATERIALS AND METHODS}

Two model systems of $\mathrm{Zx}$ epoxidation in vitro were tested using thylakoids isolated from Arabidopsis thaliana. Six week old plants grown in a $12 \mathrm{~h} / 12 \mathrm{~h}$ photoperiod at $20^{\circ} \mathrm{C}$, after $0.5 \mathrm{~h}$ dark incubation were illuminated for $30 \mathrm{~min}$ with $1500 \mu \mathrm{E} \cdot \mathrm{m}^{-2} \mathrm{~s}^{-1}$ and homogenized 2 times by $10 \mathrm{~s}$ in isolation medium $(330 \mathrm{mM}$ Sorbitol, $20 \mathrm{mM}$ Tricine, $5 \mathrm{mM}$ EGTA, $5 \mathrm{mM}$ EDTA, $10 \mathrm{mM} \mathrm{Na}_{2} \mathrm{CO}_{3}$, $\mathrm{pH}$ 7.6). After centrifugation for $5 \mathrm{~min}$ at $4000 \mathrm{rpm}$, the supernatant was discarded and residue was resuspended in resuspension medium (300 mM Sorbitol, 20 mM Hepes, $5 \mathrm{mM} \mathrm{MgCl}, 2.5 \mathrm{mM}$ EDTA, pH 7.6). At the end chlorophyll concentration in $80 \%$ acetone was measured by the method of Arnon (Arnon, 1949). The capability of $\mathrm{Zx}$ epoxidation in thylakoids isolated by above described method, was tested first for wild type of $A$. thaliana.

e-mail: dariuszlatowski@gmail.com

* Presented at the 16th International Symposium on Carotenoids, 17-22 July, 2011, Kraków, Poland

Abbreviations: Ax, antheraxanthin; MGDG, monogalactosyldiacylglycerol; $P E$, phosphatidylethanolamine; VDE, violaxanthin de-epoxidase; Vx, violaxanthin; ZE, zeaxanthin epoxidase; Zx, zeaxanthin. 


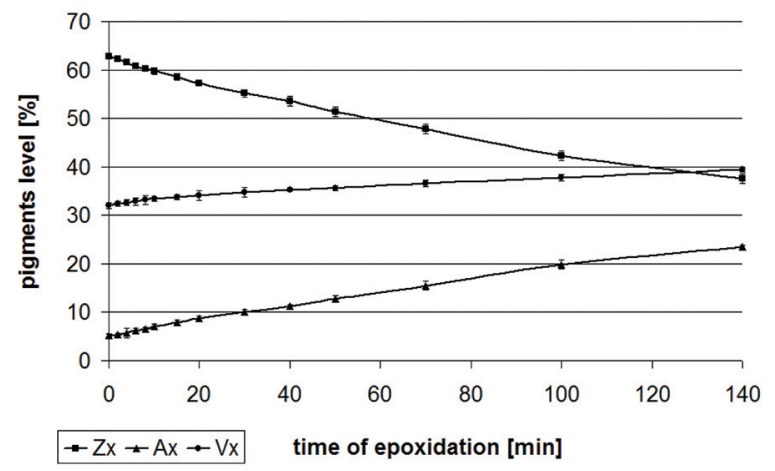

Figure 1. Kinetics of $\mathrm{Zx}$ epoxidation in thylakoids isolated from Arabidopsis thaliana wild type.

Thylakoids were sonicated for 7 seconds with 3 repeats and the amount of chlorophyll was $100 \mu \mathrm{g}$ per $\mathrm{ml}$ of reaction mixture. Zx epoxidation assay was performed for $140 \mathrm{~min}$. Ax, antheraxanthin; $\mathrm{Vx}$, violaxanthin; $\mathrm{Zx}$, zeaxanthin. The average concentrations of pigments at the beginning of epoxidation was: $V x, 14.8 \pm 0.09 ; A x$, $2.4 \pm 0.007 ; Z x, 29.0 \pm 0.029 \mathrm{nmol} / \mathrm{ml}$.

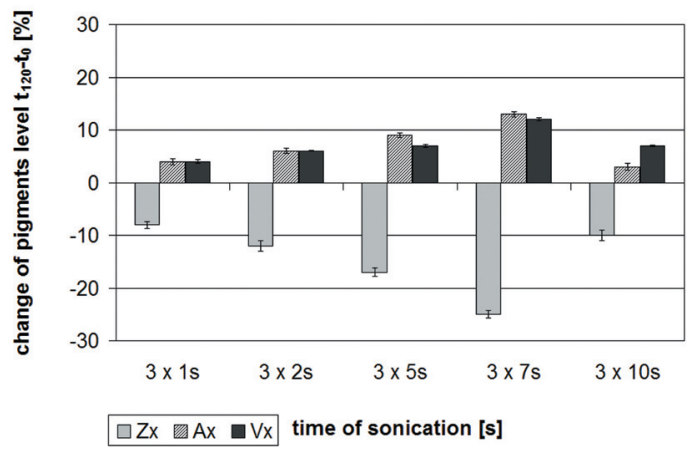

Figure 2. Change in xanthophylls level in dependence on time of sonication.

Assay system contains thylakoids isolated from npq1 and npq2 mutants and mixed in the amounts $1: 1$ and $50: 50 \mu \mathrm{g}$ of chlorophyll per $\mathrm{ml}$ of reaction mixture. Reaction was performed for 120 min. Ax, antheraxanthin; $V x$, violaxanthin; $Z x$, zeaxanthin. The average concentrations of pigments at the beginning of epoxidation was: Vx, 6.97 $\pm 0.04 ; \mathrm{Ax}, 1.16 \pm 0.01 ; \mathrm{Zx}, 20.9 \pm 0.44 \mathrm{nmol} / \mathrm{ml}$.

In the case of assay using the mutants for $\mathrm{Zx}$ epoxidation in vitro, thylakoids from two $A$. thaliana mutants (npq1 and $n p q 2$ ) were mixed together and then sonicated. The first tested parameter to develop the optimal model system for $\mathrm{Zx}$ epoxidation was the time of sonication of the $n p q 1 / n p q 2$ thylakoids mixture. Five different times of sonication were tested $(1,2,5,7,10$ seconds with 125 $\mathrm{kHz}$ and $20 \%$ amplitude) always with three repetitions. The second parameter studied in this assay system was the ratio of thylakoids from $n p q 1$ and $n p q 2$ mutants. The amount of thylakoids was determined by chlorophyll concentration. There were tested following $n p q 1$ to $n p q 2$ ratios: $1: 1(50: 50 \mu \mathrm{g} / \mathrm{ml}), 1: 1(100: 100 \mu \mathrm{g} / \mathrm{ml}), 3: 1(75: 25$ $\mu \mathrm{g} / \mathrm{ml}), 3: 1(150: 50 \mu \mathrm{g} / \mathrm{ml})$.

In another kind of model system tested, only thylakoids from $n p q 1$ mutant were used because they retain a capability of $\mathrm{Zx}$ epoxidation in vivo. In this system, instead of $n p q 2$ thylakoids as the only source of $\mathrm{Zx}$, the effect of different ratios of exogenous Zx and MGDG on epoxidation efficiency were studied. $20 \mathrm{nmol}$ of the pigment was mixed with MGDG in different proportion $(1: 15,1: 30,1: 60,1: 100)$, using methanolic stock solutions of both compounds. After mixing, the solution was evaporated by nitrogen and then the dry residue was dis-

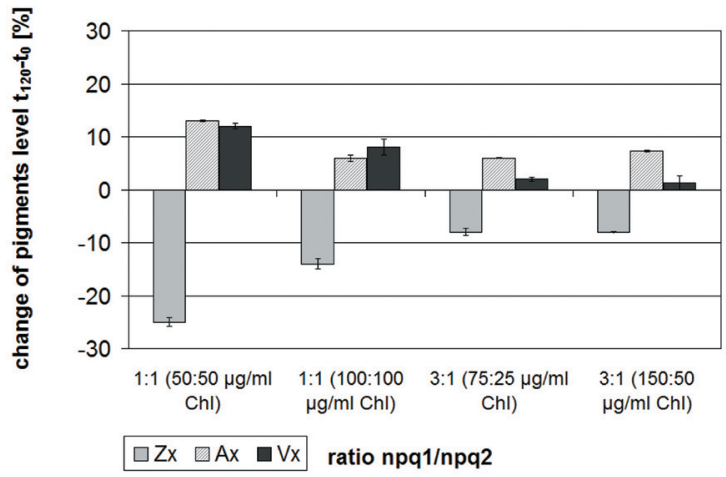

Figure 3. Change in xanthophylls level dependent on the ratio of thylakoids from both mutants.

Thylakoids were sonicated for 7 seconds with three repeats. Reaction was performed for $120 \mathrm{~min}$. Ax, antheraxanthin; Chl, chlorophyll; Vx,- violaxanthin; Zx, zeaxanthin. The average concentrations of pigments at the beginning of epoxidation was: for 1:1 $(50: 50 \mu \mathrm{g} / \mathrm{ml}) \mathrm{Vx}-6.97 \pm 0.04, A x-1.16 \pm 0.01, \mathrm{Zx}-$ $20.9 \pm 0.44 \mathrm{nmol} / \mathrm{ml}$; for $1: 1(100: 100 \mu \mathrm{g} / \mathrm{ml}) \mathrm{Vx}-13.4 \pm 0.08$, Ax - 2.23 $\pm 0.002, \mathrm{Zx}-40.2 \pm 0.84 \mathrm{nmol} / \mathrm{ml}$; for $3: 1(75: 25 \mu \mathrm{g} / \mathrm{ml}) \mathrm{Vx}$ - 4.63 $\pm 0.05, \mathrm{Ax}-0.56 \pm 0.003, \mathrm{Zx}-6.1 \pm 0.02 \mathrm{nmol} / \mathrm{ml}$; for $3: 1$ $(150: 50 \mu \mathrm{g} / \mathrm{ml}) \mathrm{Vx}-7.33 \pm 0.15, \mathrm{Ax}-0.9 \pm 0.003, \mathrm{Zx}-10.5 \pm 0.05$ $\mathrm{nmol} / \mathrm{ml}$.

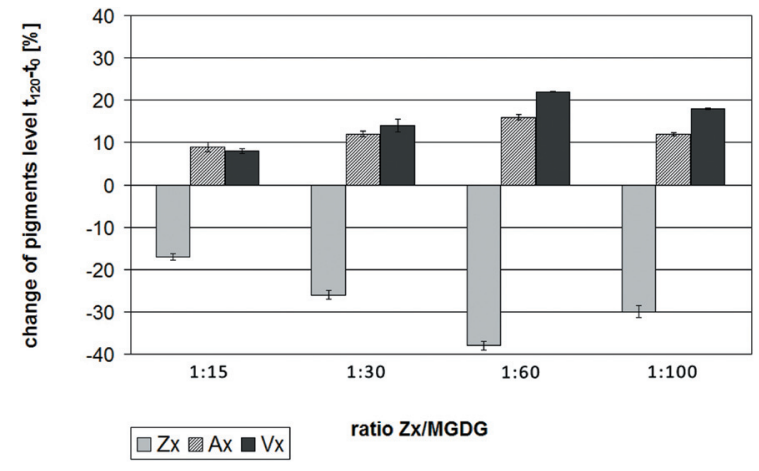

Figure 4. Change in xanthophylls level dependent on the ratio of zeaxanthin and MGDG.

Ethanolic solution of both compounds was added into reaction mixture. Reaction was performed for $120 \mathrm{~min}$. Ax, antheraxanthin; MGDG, monogalactosyldiacylglycerol; Vx, violaxanthin; Zx, zeaxanthin. The concentration of exogenous $Z x$ at the beginning of the epoxidation was $20 \mathrm{nmol} / \mathrm{ml}$.

solved in ethanol. This solution was added into reaction mixture. The concentration of ethanol in the reaction medium was less than $2 \%$. Epoxidation reaction was performed for 120 minutes at room temperature and the level of three pigments $(\mathrm{Zx}, \mathrm{Vx}$, antheraxanthin $(\mathrm{Ax})$ ) were measured by HPLC as described earlier (Latowski et al., 2002). The reaction mixture contained: thylakoids, epoxidation medium (400 mM Sorbitol, $50 \mathrm{mM}$ Hepes, 5 $\mathrm{mM} \mathrm{MgCl}, \mathrm{pH} \mathrm{7,6)}$, albumin, sodium ascorbate, FAD, NADPH.

\section{RESULTS AND DISCUSSION}

The studies on Zx epoxidation in in vitro systems were not possible because up today the enzyme involved in this reaction, zeaxanthin epoxidase, has not been isolated and no model system for analysis of kinetics or molecular mechanism of this reaction has been developed. It was also difficult to study of Zx epoxidation in isolated thylakoids although two methods for thylakoids isolation 
where ZE was working were described for plants. One of them was for lettuce (Siefermann \& Yamamoto, 1974) and the other for spinach (Büch et al., 1995). The obtained results show that our method of thylakoids isolation from model plant, $A$. thaliana, which is presented in this work, also permits to study Zx epoxidation in isolated thylakoids (Fig. 1). In fact the presented method uses a fraction containing fragmented chloroplasts with thylakoids and stroma and therefore ZE, which is located at the stromal side of thylakoids, is also present in the final suspension. In assay system using thylakoids from $n p q 1$ and $n p q 2$ mutants the optimal time of their sonication was defined as 7 seconds with $125 \mathrm{kHz}$ and $20 \%$ amplitude with 3 repeats. It was observed that after that time of sonication, epoxidation is the most efficient (Fig. 2). Probably such time of sonication allow better fusion of the two types of thylakoids and, on the other hand, it does not cause significant destruction of isolated structures. The second parameter studied in this model system was the ratio of thylakoids from $n p q 1$ and $n p q 2$. The optimal ratio was estimated as 1:1 (Fig. 3). The amount of thylakoids in reaction mixture was also important for Zx epoxidation efficiency. The optimum was defined as $100 \mu \mathrm{g}$ of chlorophylls per $1 \mathrm{ml}$ of epoxidation mixture. Higher concentration is not advantageous. In this model system with optimal proportion of the thylakoids from both mutants and optimal sonication conditions the amount of Zx was reduced by $25 \%$ (Fig. 3).

The most simplified system we studied consists of $n p q 1$ mutant thylakoids supplemented with exogenous Zx with MGDG in varying proportions. The optimal ratio of $Z x$ to MGDG was defined as 1:60. The low amount of ethanol added to the assay mixture with $\mathrm{Zx}$ and MGDG did not reduce the efficiency of the reaction. In optimal conditions the amount of $\mathrm{Zx}$ was reduced by $38 \%$ (Fig. 4). The in vitro assay with exogenous $\mathrm{Zx}$, is a very promising model system permitting studies on the $\mathrm{Zx}$ epoxidation. The use of exogenous substrate allows better controlling the reaction conditions and provides new possibilities to investigate molecular mechanism of epoxidation in the xanthophylls cycle.

\section{Acknowledgements}

This work was supported by the Polish Ministry of Science and Higher Education (project No. 50/NDFG $/ 2007 / 0$ ) and the German Science foundation (DFG project JA-665/8-1).

\section{REFERENCES}

Arnon DI (1949) Copper enzymes in isolated chloroplasts. Polyphenoloxidase in Beta vulgaris. Plant Physiol 24: 1-15.

K. Büch, H. Stransky, A. Hager (1995) FAD is a further essential cofactor of the $\mathrm{NAD}(\mathrm{P}) \mathrm{H}$ and $\mathrm{O}_{2}$-dependent zeaxanthin epoxidase. FEBS Letters 376: 45-48.

Garcia-Plazaola JI, Matsubara S, Osmond CB (2007) The lutein epoxide cycle in higher plants: its relationships to other xanthophyll cycles and possible functions. Funct Plant Biol 34: 759-773.

Goss R, Lepetit B, Wilhelm C (2006) Evidence for a rebinding of antheraxanthin to the light-harvesting complex during the epoxidation reaction of the violaxanthin cycle. J. Plant Physiol 163: 585-590.

Hager A, Holocher K (1994) Localization of the xanthophyll-cycle enzyme violaxanthin de-epoxidase within the thylakoid lumen and abolition of its mobility by a (light-dependent) $\mathrm{pH}$ decrease. Planta 192: 581-589.

Havaux M, Bonfils JP, Lütz C, Niyogi KK (2000) Photodamage of the photosynthetic apparatus and its dependence on the leaf developmental stage in the $n p q 1$ Arabidopsis mutant deficient in the xanthophyll cycle enzyme violaxanthin deepoxidase. Plant Physiol 124: 273-284.

Jahns P, Latowski D, Strzałka K (2009) Mechanism and regulation of the violaxanthin cycle: the role of antenna proteins and membrane lipids. Biochim Biophys Acta 1787: 3-14.

Latowski D, Åkerlund HE, Strzałka K (2004) Violaxanthin de-epoxidase, the xanthophyll cycle enzyme, requires lipid inverted hexagonal structures for its activity. Biochemistry 43: 4417-4420.

Latowski D, Kruk J, Burda K, Skrzynecka-Jaskier M, Kostecka-Gugała A, Strzałka K (2002) Kinetics of violaxanthin de-epoxidation by violaxanthin de-epoxidase, a xanthophyll cycle enzyme, is regulated by membrane fluidity in model lipid bilayers. Eur J Biochem 269: 4656-4665.

Siefermann D, Yamamoto HY (1975) Light-induced de-epoxidation of violaxanthin in lettuce chloroplasts IV. The effects of electrontransport conditions on violaxanthin availability. Biochim Biophys Acta 387: 149-58.

Yamamoto HY, Higashi RM (1978) Violaxanthin de-epoxidase. Lipid composition and substrate specificity. Arch Biochem Biophys 190: 514 522. 